\title{
Implementation of Formation Transition System using Synchronization in a Mobile Robot Group
}

Takaaki Shimone $^{1}$, Daisuke Kurabayashi ${ }^{2}$, Kunio Okita $^{3}$, and Tetsuro Funato $^{2}$

1 Kansai Electric Power Inc. shimone@irs.ctrl.titech.ac.jp

2 Tokyo Institute of Technology \{dkura, funato\}@irs.ctrl.titech.ac.jp

3 RICOH co., ltd.

Summary. This study proposes an algorithm for the passage of a mobile robot group through a narrow space. We deal with a robot group composed of one leader and multiple unlabeled followers. A steady formation is realized by generating a Delaunay diagram using the profiles of surrounding robots. Further, we resolved a deadlock situation by using the phase gap of nonlinear oscillators to which the profiles of the surrounding obstacles are provided as inputs. We implement the proposed system in a real multi-robot system in order to demonstrate its effectiveness.

\section{Introduction}

In a system comprising several robots, it is effective to arrange them in a formation in order to facilitate their navigation. In such systems, it is sufficient to control (or assign a desired path to) a leader robot. Other followers maintain their appropriate relative positions and follow the leader to reach their destination. There exist several proposals regarding grouping and maintaining formation control.

Das et al. have proposed an elegant control algorithm of formation control among multiple vehicles by using omni-direction camera [1]. They have discussed design of possible controllers under description of a directed graph among labeled robots. They also have proposed formation switching algorithm under the order of labeled robot.

In this paper, we are going to deal with a group of anonymous, unlabeled robots. For the fault-tolerance of a robot group, considering breakdowns of some members in a group, we need to consider such unlabeled robots. Especially, we focus on solving deadlock caused by geometrical features of environment. One of the most common system to navigate unlabeled robot group is potential method [2] [3]. However, when a robot group encounters obstacles, the two objectives of maintaining a formation and avoiding obstacles 
often conflict with each other. A deadlock situation is often caused by the symmetric features of the environment around a robot group (Fig.1).

Here, we propose and implement an algorithm that is compatible with both maintaining a formation and resolving deadlocks. For the former, we employ a Delaunay diagram generated using the profiles of surrounding robots. A geometrically steady formation is maintained by acquiring the best adjacent relation from this diagram. For the latter, we employ nonlinear oscillators that input the profiles of surrounding obstacles. Deadlocks are resolved by using the phase gaps of nonlinear oscillators that transit in different states. We have implemented simple synchronization system, which is also utilized to distinguish robots from obstacles.

\section{Model of a robot group}

\subsection{Problem settlement}

In this research, a robot group is composed of a single high-performance (or manually operated) leader and multiple followers that follow the leader.

We assume that all the robots have the following common characteristics:

- The shape of a robot is a circle of radius $R$.

- The robots can move in omni direction.

- The robots can measure the relative position of an object within their constant range (sight range).

- The robots can acquire the internal state of the nonlinear oscillator of other robot.

The objective of this research is that the leader should guide all the followers to the destination in an environment with obstacles, as shown in Fig.2.

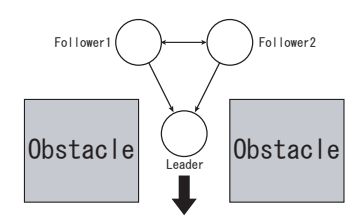

Fig. 1. Example of a deadlock situation for a robot group

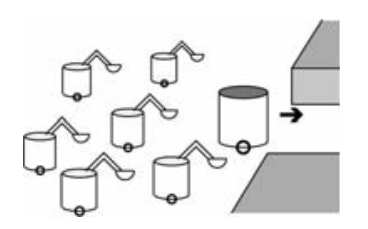

Fig. 2. Sketch of a robot group that is entering a narrow corridor guided by a leader

\subsection{Formulation of followers}

We assume that a robot group is led by a leader. The robots in this group move according to a simple system (1) [4]. 


$$
{ }^{i} \dot{x}=\sum_{j \in \mathrm{NBR}_{\mathrm{i}}} \rho_{i j} \tau \frac{{ }^{i} x_{j}}{\left|{ }^{i} x_{j}\right|}+\sum_{j \in \mathrm{OBJECTS}_{\mathrm{i}}} \delta \frac{{ }^{i} \tilde{x}_{j}}{\left|{ }^{i} \tilde{x}_{j}\right|}
$$

where ${ }^{i} \dot{x}$ and ${ }^{i} x_{j}$ represent the relative velocity of robot $i$ and its distance from robot $j$, respectively, in terms of the coordinates of robot $i ;{ }^{i} \tilde{x}_{j}$ represents the relative distance between the nearest point of object $j$ and the center of $i ; \tau$ and $\delta$ are constants; $\rho_{i j}$ represents the strength of the robot linkage between $i$ and $j ; \mathrm{NBR}_{\mathrm{i}}$ represents a set of the neighboring robots; and OBJECTS represents a set of visible objects.

In this research, we implement a robot formation by obtaining $\mathrm{NBR}_{\mathrm{i}}$ autonomously. Furthermore, a deadlock situation is resolved by adjusting the balance of $\rho_{i j}$.

\subsection{Finding appropriate neighbors}

We employ Delaunay diagram so that each robot can determine its neighboring robots autonomously. The Delaunay diagram is a graph in which a natural adjacent relation is derived from a geometrical one. We can generate this diagram from the profiles of the surrounding robots. The neighboring robots of a given robot are the ones adjacent to it, as shown in Fig. 3.

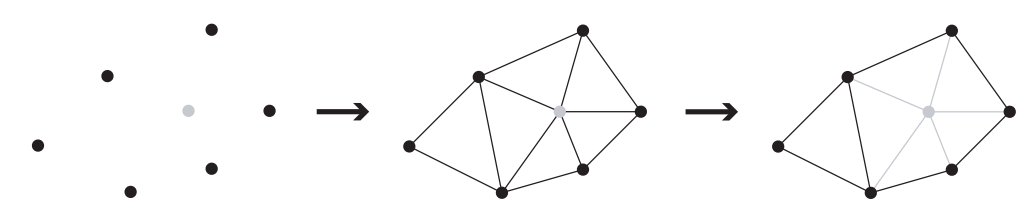

Fig. 3. Determination of neighboring robots

\section{Resolving deadlock by formation switching}

\subsection{Conceptual design by using oscillators}

We employ van der Pol (VDP) oscillator [5], which is a well-known nonlinear oscillator. The dynamics of this oscillator can be expressed as (2),

$$
\ddot{p}_{i}-\varepsilon\left(1-p_{i}^{2}\right) \dot{p}_{i}+\omega^{2} p_{i}=0
$$

where $p_{i}, \varepsilon$, and $\omega$ indicate the state variable of oscillator $i$, the nonlinearity, and the eigen frequency, respectively. Typically, connected VDP oscillators can synchronize with each other. We formulate the connected oscillators by using (3): 


$$
p_{i}(t+1) \leftarrow p_{i}(t+1)+\frac{1}{\left|N_{i}\right|} \sum_{j \in N_{i}} \gamma_{j}\left(p_{j}(t)-p_{i}(t)\right)
$$

where $N(i)$ and $\gamma_{j}$ represent the assembly of connected oscillators, and the gain of interactions among other oscillators, respectively.

The VDP oscillator can synchronize with a periodic external input similar to the synchronization with other VDP oscillators. Let us introduce an external input $F_{i}$ as follows:

$$
\ddot{p}_{i}-\varepsilon\left(1-p_{i}^{2}\right) \dot{p}_{i}+\omega^{2} p_{i}=F_{i}
$$

Here, let us suppose quite simple expression: $F_{i}=A_{i} \cos \left(\Omega t+\phi_{i}\right)$. Assuming that $\omega \approx \Omega$, VDP oscillator synchronizes with an external input. This VDP oscillator exhibits a response dissimilar to that of other oscillators that have not synchronized with an external input.

We use these characteristics of VDP oscillator. We implement VDP oscillator in each robot. By communicating each other, VDP oscillators make interactions, which may synchronize them. Without external input, the VDP oscillator of each robot is synchronized with others. In this case, phase gaps among oscillators becomes quite small. By arranging linkage among robots according to the phase gap, we can make a formation of robot tight. On the other hand, by generating external signals according to detected obstacles, they receive a forcing oscillation and correspondingly converge into different states. Then, the phase gaps becomes larger and unequal. Thus, we can expect resolving a deadlock situation by adjusting the balance of the strength of robot linkage corresponding to the phase gaps. By the concept, when passing through a narrow space, a robot group undergoes formation transition by acquiring parameter $\gamma_{j}$ corresponding to the environment.

\subsection{Sensor signals: periodic external input}

We propose a method for generating an external input from the profiles of the surrounding obstacles. We assume that the robots are equipped with a LASER distance scanner that can measure the relative position of the object within a certain sight range. In reality, this sensor rotates a LASER beam and produces a profile of the area around the robot, as shown in Fig.4. Because the sensor makes scanning in certain period, which is very much faster than the speed of robot motion, we regard that the obtained series of data is a periodic signal. If we extract characteristics of the sensor signal in a specific range of frequency, the VDP may synchronize with the external input, so that synchronization among VDPs may be disturbed. Because objects far from a robot does not disturb it, we translate measured distance by robot $i, \hat{d}_{i}$, into $d_{i}$ as follows:

$$
d_{i}\left(\theta_{i}\right) \propto \frac{1}{\hat{d}_{i}\left(\theta_{i}\right)}
$$




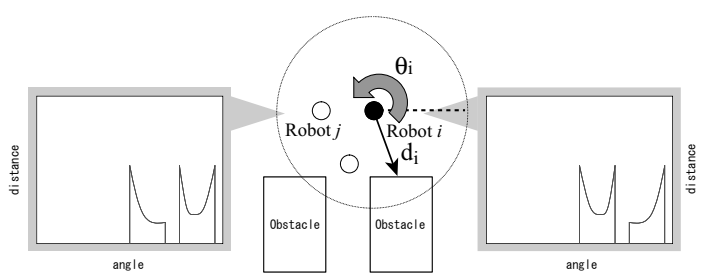

Fig. 4. Profile scanning of surroundings

Fig. 4 illustrates schematic view of sensing. Robot $i$ and $j$ obtain profiles of surroundings as left and right graph, respectively. Even if they are in symmetric positions, the generated graphs are different. Thus, even in a deadlock caused by symmetric features, followers can generate an asymmetric signal. However, the output of the scanning sensor may contain high frequency signals that cannot be modulated by the oscillator. We apply the discrete Fourier transformation [6] to extract the dominant property. Then, we simplify the sensor output as $(6)$ :

$$
F_{i}=\left|c_{1}\right| \cos \left(\omega t+\phi_{1}\right)
$$

where $\left|c_{n}\right|$ and $\phi_{n}$ represent the size and phase of the Fourier coefficient, respectively.

\subsection{Mapping of $\rho_{i j}$}

We change the strength of the robot linkage $\rho_{i j}$ according to the phase gap $\theta_{i j}$ between $i$ and $j$. Thus, mapping from the phase gaps to $\rho_{i j}$ is required. Let us consider the ideal formation transition, shown in Fig. 5, when passing through a narrow space. This phenomenon is caused by the decrease in the strength of the robot linkage with the increase in distance. To realize the above mentioned phenomenon, we calculate $\gamma_{j}$ using (7). This calculated parameter can reduce the phase gap when the distance of the robot linkage increases. Finally, the phase gap is mapped to the strength of robot linkage by increasing monotonically.

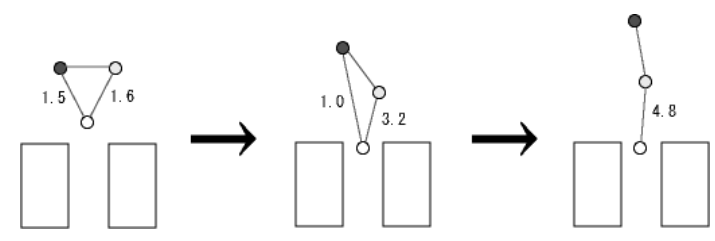

Fig. 5. Schematic view of formation transition 


$$
\gamma_{j}\left(D_{i j}\right)=\gamma_{0}+\frac{\gamma_{u p}}{1+e^{-\alpha\left(D_{i j}-\theta_{1}\right)}}+\frac{\gamma_{u p}}{1+e^{-\alpha\left(D_{i j}-\theta_{2}\right)}}
$$

where $\gamma_{0}$ and $\gamma_{u p}$ are certain statics. Then, we set the mapping of $\rho_{i j}$ as (8).

$$
\rho_{i j}\left(\left|\theta_{i j}\right|\right)=K\left|\theta_{i j}\right|+1
$$

where $K$ is a fixed parameter. To determine $K$, we have carried out a heuristic optimization considering the time required to resolve a default deadlock situation. In this study, we define two default deadlock situations, as shown in Fig.6(a) and (b). We search for a valid range of $K$ that can be used for mapping by adjusting its value. Accordingly, the following results are obtained: $11.5 \leq K \leq 28.5$ and $12.0 \leq K \leq 30.0$. We adopt a common region of the abovementioned ranges $* 12.0 \leq K \leq 28.5)$ as the meaning solution as shown in Fig.6(c).

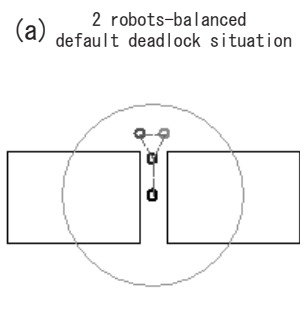

3 robots-balanced
(b) default deadlock situation

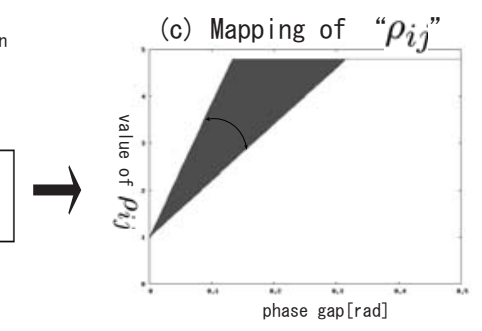

Fig. 6. Valid range of parameter K

\subsection{Simulation of a robot group}

We conducted a computer simulation to verify the transitions of the phase gaps and the movements of the robot group. Table 1 lists the simulation parameters.

Table 1. Simulation parameters

\begin{tabular}{|c|c|c|c|c|c|}
\hline Radius & View Radius & $\tau$ & $\delta$ & $K$ & Range of narrow space \\
\hline 0.3 & 5.0 & 50 & 300 & 12.0 & 1.5 \\
\hline
\end{tabular}

Fig.7 illustrates the transitions of the group formation comprising five robots. Based on the transitions of $\rho_{i j}$ shown in Fig.8, we can state that the proposed algorithm changes the gain successfully in order to maintain the formation. The robots modified their formation according to the environment. 


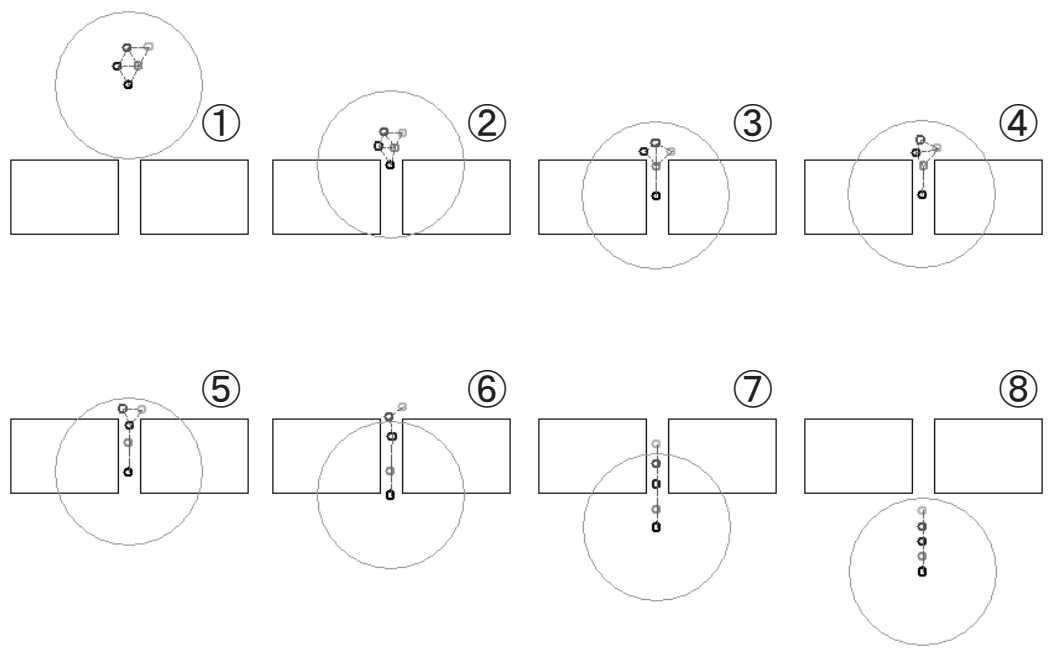

Fig. 7. Formation transition in simulation

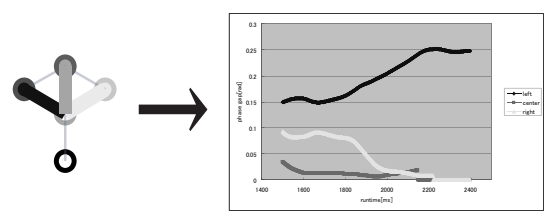

(a) 3 robots-balanced default deadlock situation (as shown in (3) (5))

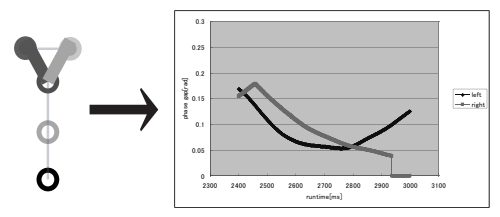

(b) 2 robots-balanced default deadlock situation (as shown in (5) (6))

Fig. 8. Transitions of $\rho_{i j}$ (gain) to maintain a formation

\section{Implementation}

\subsection{Robot platform}

The robot used as both leader and follower is shown in Fig.9. This two-wheeled robot is based on e-puck, small autonomous mobile robot base [7]. The shape of this robot is a cylinder of $8.5[\mathrm{~cm}]$ in the diameter. The robot is equipped with a small micro computer, dsPIC. This unit can calculate the amount of robotic movement and state of an oscillator in real time.

A laser-scanning distance sensor URG-04LX [8] is attached on the top of the robot. This sensor composes a laser source and rotating mirror, and it can measure a distance of approximately 240 [deg] using the time-of-flight of the laser ray. Using this sensor, the robot acquires the relative position of other robots for formation maintenance and cyclic signal for external input to the oscillator. 

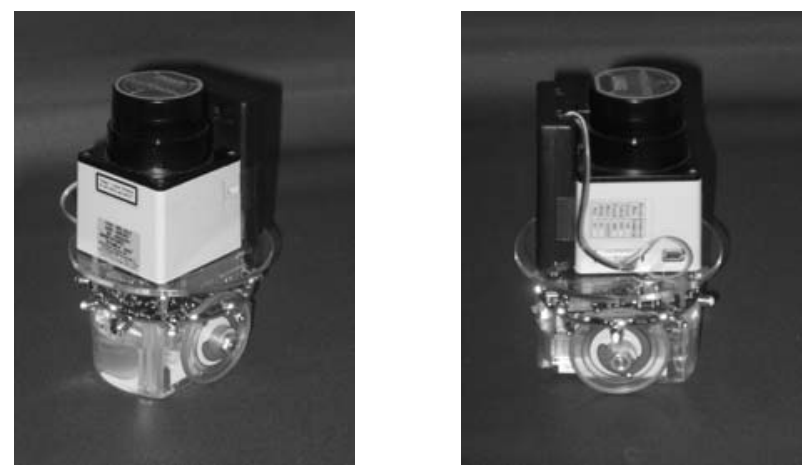

Fig. 9. The two wheeled robot

\subsection{Synchronization of the oscillators}

The robot is equipped with light-emitting elements composed of LED units and light-sensitive elements of phototransistors in eight directions. All lightemitting elements flash when the phase of the oscillator becomes $0[\mathrm{deg}]$. Using its light-sensitive elements, the robot can acquire the timing of phase 0 of another robot by detecting flash of another robot. As a result, the robot can estimate phase $\theta_{j}$ of other robots at any instance because the cycle of all the oscillators is constant in this study. The limit cycle of the oscillator can be approximated to an elliptic orbit as follows:

$$
\frac{p^{2}}{4}+\frac{\dot{p}^{2}}{4 \omega^{2}}=1
$$

By introducing $\theta_{j}$ in (9), we obtain the internal state of another robot $p_{j}$ as follows:

$$
p_{j}=2 \cos \left(\arctan \left(\frac{\tan \theta_{j}}{\omega}\right)\right)
$$

By introducing $p_{j}$ in (3), we implement the synchronization of the oscillators.

By using this system, we verified the synchronization and transition to an asynchronous state of oscillators in the real robots. The experimental setup is shown in Fig. 10. We position three robots with differing initial phases, and started synchronization procedure. Once the synchronization confirmed, we place a narrow space (obstacle) in front of the leader in order to observe the phase gaps between the followers' oscillators.

Fig. 11(a) and (b) shows the snapshots and the time schedule of the experiment, respectively. Based on these results, we can see that the differing initial phases are synchronized by the estimated calculation and that the three oscillators generate a phase gap when the obstacle is introduced. 


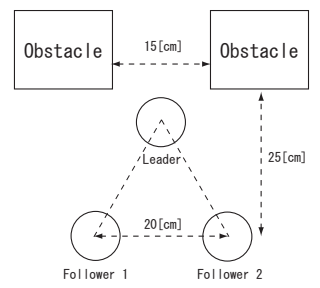

Fig. 10. The experimental setup
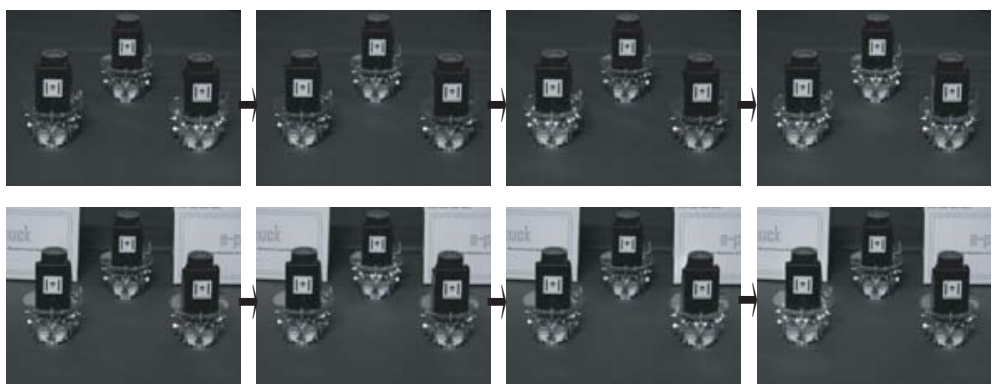

(a) Verification of synchronization and transition to asynchronous system

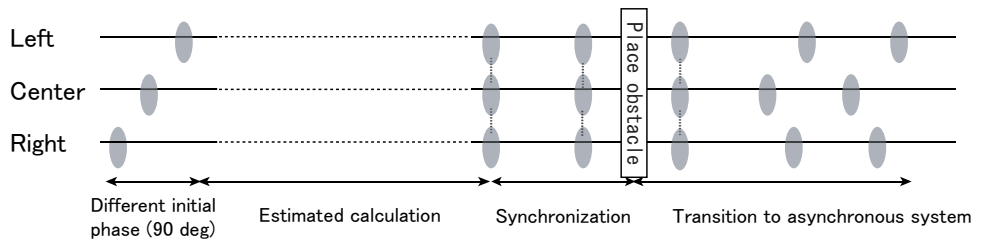

(b) Time schedule

Fig. 11. The experiment of synchronization

\subsection{Formation transition}

Finally, we conducted an experiment using three robots. One of them is a leader that goes directly to the destination, while the other are followers . Fig. 12 shows the snapshots of the experiment. By using the proposed algorithm and system, the two followers successfully modified their triangular formation into a linear one in order to pass through a narrow space.

\section{Conclusions}

In this paper, we proposed a formation transition algorithm to adapt to the geometrical features of an environment. We consider a robot group composed of one leader and multiple unlabeled followers. We employed Delaunay diagram to maintain a geometrically steady formation and nonlinear oscillators 
(1)

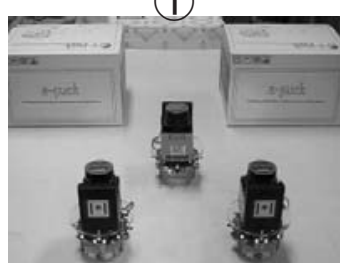

(4)

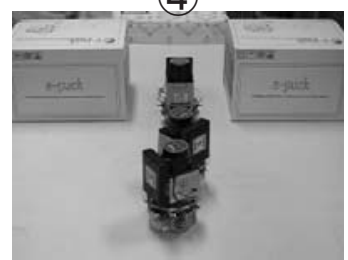

(2)

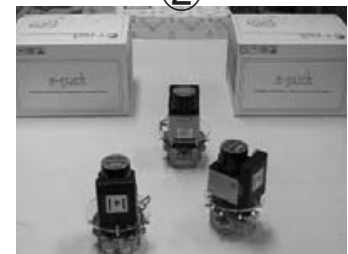

(5)

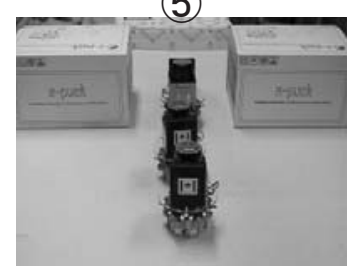

(3)

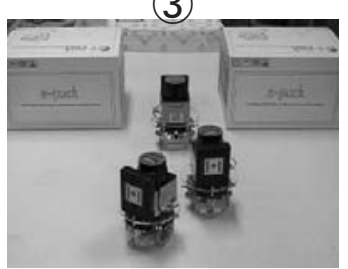

(6)

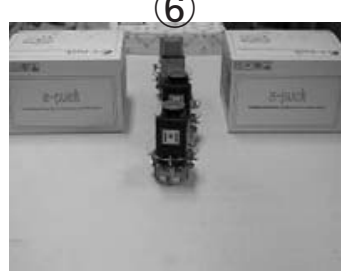

Fig. 12. Result of the experiment involving three robots

to avoid deadlocks in a situation involving symmetric features. We verified the effectiveness of the algorithm by simulations and experiments.

\section{Acknowledgement}

This research is partially supported by Grants-in-Aid for Scientific Research, MEXT, Japan, under contract, 17075007, and 17680016.

\section{References}

1. Das A K, Fierro R, Kumar V, Ostrowski J P, Spletzer J, Taylor C J (2002) A Vision-Based Formation Control Framework, IEEE Trans. Robotics and Automat., 18:813-825

2. Ota J, Arai T (1993) Motion Planning of Multiple Mobile Robots Using Dynamic Groups, Proc.1993 IEEE Int. Conf. Robotics and Automat., 28-33

3. Ota J, Arai T, Yokogawa Y (1994) Distributed Strategy-Making Method in Multiple Mobile Robot System, Distributed Autonomous Robotics Systems, 103-106

4. Yamaguchi H (1998) A Cooperative Hunting Behavior by Mobile Robot Troops , Proc.1998 IEEE, Robotics and Automat., 3204-3209

5. Shimizu M, Ishiguro A, Kawakatsu T, Masubuchi Y, Doi M (2003) Coherent Swarming from Local Interaction by Exploiting Molecular Dynamics and Stokesian Dynamics Methods, Proc. Int. Conf. Intelligent Robots and Systems, 16041619

6. Tolimieri R, An M, Lu C (1997) Algorithms for Discrete Fourier Transform and Convolution (Signal Processing and Digital Filtering), Sprinver-Verlag

7. EPFL education robot, http://www.e-puck.org/

8. Hokuyo Automatic, http://www.hokuyo-aut.co.jp/02sensor/07scanner/urg.html 